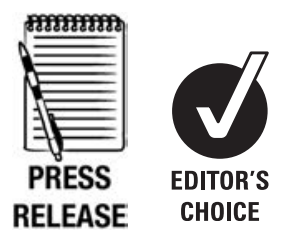
Medicine, Addenbrookes Hospital, Cambridge University Hospitals NHS Trust, Cambridge, UK

${ }^{2}$ Department of Cytology, Addenbrookes Hospital, Cambridge University Hospitals NHS Trust, Cambridge, UK ${ }^{3}$ Centre for Applied Medical Statistics, University of Cambridge, Cambridge, UK

\section{Correspondence to} Dr Christopher A Carne, Department of Genitourinary Medicine, Clinic 1A, Box 38, Addenbrookes Hospital, Cambridge University Hospitals NHS Trust, Cambridge CB2 000, UK; christopher.carne@ addenbrookes.nhs.uk

Accepted 6 April 2012

Published Online First 6 December 2012

\title{
Low sperm counts in genitourinary medicine clinic attendees: results from a case-control study
}

\author{
Christopher A Carne, ${ }^{1}$ Sian Chilcott, ${ }^{2}$ Christopher Palmer, ${ }^{3}$ Oliver Green, ${ }^{2}$ \\ Simeon Bridge, ${ }^{1}$ Richard Walsh, ${ }^{2}$ Anna Gramy-Mason, ${ }^{1}$ Maria 0'Donovan ${ }^{2}$
}

\begin{abstract}
Objectives To conduct a case-control study of abnormalities in the semen of genitourinary (GU) medicine clinic attendees compared with general practice (GP) controls and in patients with asymptomatic and symptomatic non-specific urethritis (NSU) before and after the urethritis resolves.

Methods Rates of semen abnormalities were compared between the different groups (19 with symptomatic and 27 with asymptomatic NSU, seven with symptomatic non-NSU and 64 clinic controls) and between clinic attendees and 417 patients attending GP for the first investigation of possible infertility. Those with symptomatic or asymptomatic NSU gave repeat semen samples on resolution of the NSU.
\end{abstract}

Results The study included 117 clinic volunteers. They were shown to have statistically significantly worse total sperm counts $(p=0.002)$, volume of semen $(p<0.001)$ and percentage of abnormal forms $(p<0.04)$ compared with $417 \mathrm{GP}$ controls. Compared with the rest of the clinic volunteers, asymptomatic NSU patients had statistically significantly lower total sperm counts $(p<0.02)$. Asymptomatic NSU patients had statistically significantly lower total sperm counts compared with symptomatic NSU patients $(p<0.02)$. Compared with GP controls, clinic controls had statistically significantly inferior total sperm counts $(p=0.009)$ and semen volume $(p<0.001)$.

Conclusions GU clinic attendees are more likely to have abnormalities of semen than patients attending GP for the first check for possible infertility. A high rate of abnormal semen findings are found in patients with and without NSU but the highest rate occurred in those with asymptomatic NSU. Is asymptomatic NSU therefore pathogenic and does it require treatment like symptomatic NSU?

\section{INTRODUCTION}

In developed countries, $22 \%$ of infertility is caused by a male factor alone and in a further $21 \%$ both male and female factors are involved. ${ }^{1}$ Subfertility affects one in 20 men. $^{2}$ Known causes of male subfertility are genital tract infection (epididymitis and orchitis), testicular disease and abnormalities, systemic disease and external factors, for example, drugs. $^{2}$ Male subfertility is rarely caused by endocrine deficiency. ${ }^{2}$ The evidence linking environmental factors and impaired human fertility is weak. $^{3}$

Epididymitis, a recognised cause of male infertility, $^{2}$ is a common complication of urethritis. In genitourinary (GU) medicine clinics in the UK, cases of non-chlamydial/non-gonococcal (nonspecific) epididymitis are seven times as common as those associated with Chlamydia trachomatis. ${ }^{4}$ It is well recognised that $C$ trachomatis infection in women can cause infertility following a symptomatic pelvic infection or asymptomatically without a clinically apparent salpingitis. ${ }^{5}$ Although it is known that symptomatic epididymitis can cause male infertility, ${ }^{2}$ there is no clear in vivo evidence that asymptomatic non-chlamydial urogenital infection can have an adverse effect on male fertility. ${ }^{6}$

The findings of an epidemiological study suggested an association between infection with $C$ trachomatis (CT) in men and unexplained infertility and implied that infection was frequently asymptomatic. ${ }^{7}$ CT has been found to be asymptomatic in around $50 \%$ or more cases in community and clinic settings in the UK and in a rural setting in Tanzania. ${ }^{8-10}$

In vitro work suggests that male genital tract infections may adversely affect sperm quality and fertilising potential by a variety of mechanisms, for example, infection-related impairment of accessory gland function, partial or full obstruction of sperm transport, infection-related disruption of the bloodtestis barrier inducing the generation of anti-sperm antibodies, and so on. ${ }^{11}$

The diagnosis of non-specific urethritis (NSU) (non-chlamydial and non-gonococcal) is made on microscopy. The latest guideline from the British Association for Sexual Health and HIV has changed former guidance so that screening for asymptomatic NSU and checking for unresolved asymptomatic NSU after treatment of symptomatic NSU are no longer advocated. ${ }^{12}$ However, other clinicians have advocated caution in adopting such policies before further research has been done. ${ }^{13}$ This study examines fertility in men with NSU, that is, negative for both Neisseria gonorrhoeae (NG) and CT, and other clinic attendees. Primary aims were to investigate whether there is a higher incidence of abnormalities in the semen of men with urethritis compared with controls and second to investigate whether any abnormalities found in the semen of men with urethritis are corrected when the urethritis resolves. A secondary aim was to investigate whether asymptomatic urethritis has similar effects (if any) on semen to symptomatic urethritis. The hypothesis was that just as infertility may be caused by either symptomatic or asymptomatic infection in women, so the same may occur in men. 


\section{METHODS}

\section{Study population}

Male genitourinary clinic attendees aged 16 years and over were invited to participate in the study. They were recruited between March 2006 and February 2010 and were required to give signed written consent. Follow-up finished in September 2010. Ethics approval was granted by the Peterborough and Fenland Local Research Ethics Committee. The study took place in the Department of Genitourinary Medicine at Addenbrookes Hospital.

Inclusion criteria were patients who had ejaculated at some time in the previous week but not in the $48 \mathrm{~h}$ before the study sample and had held their urine for at least $3 \mathrm{~h}$. Exclusion criteria were those aged under 16 years and patients found on questionnaire to have a known or suggested cause of male infertility.

These comprised prescription and recreational drugs known to cause male infertility, a history of testicular disease or abnormality or intrascrotal surgery, epididymitis or orchitis, or influenza or other serious illness in the previous 3 months. As patients with symptoms suggestive of possible prostatitis were not included, neither prostatitis questionnaires nor MearesStamey tests were used. Patients found to have $C$ trachomatis were excluded from the analysis.

Patients were defined as having urethritis based on the analysis of a urethral smear according to the Giessen urethritis protocol, where there is evidence of $>4$ granulocytes per microscopic field $(1000 \times) .{ }^{14}$ Patients were divided into symptomatic (dysuria and/or urethral discharge) or asymptomatic (entirely free of symptoms). Clinic controls were asymptomatic men seeking a sexual health screen whose urethral smear showed that they did not have urethritis. It proved impossible to recruit a community control group. Therefore, a comparator group was formed from data collected by the Addenbrookes Hospital Cytology department on 417 patients attending general practice (GP) requesting a first check for possible infertility between January and October 2006 (referred to as GP controls). The same eligibility criteria for semen analysis applied, although this could not be checked by the study authors. A further small group comprised patients with dysuria and/or discharge who were negative for NGU on an early morning smear.

\section{Tests and questionnaire}

Patients and clinic controls were required to hold their urine for at least $3 \mathrm{~h}$ before the urethra was swabbed. The study started before first pass urine samples had largely supplanted urethral swabs for chlamydia.

Each participant was required to answer questions to enable the doctor to complete a research questionnaire covering known and suggested determinants of male factor infertility ${ }^{2}$ Patients found on questionnaire to have a known or suggested cause of male infertility were excluded (see Introduction section).

Participants also underwent a screen for sexually transmitted infections as indicated by their clinical presentation and wishes. These tests included a urethral smear (as above), a urethral swab for culture for $N$ gonorrhoeae and a urethral swab for PCR for $C$ trachomatis.

Participants were also invited to produce a semen sample in the clinic after the consultation prior to starting their course of antibiotics (if appropriate). All patients found to have NGU were treated with Azithromycin $1 \mathrm{~g}$ oral stat. All patients were asked to return at 2 weeks to receive the results of their tests. Those who had presented with NGU had a repeat urethral smear for quantitation of pus cells. Those with NSU also gave a repeat semen sample on resolution of their urethritis. Semen analysis was conducted according to the routine protocol. ${ }^{15}$ NSU patients were also invited to return $\geq 3$ months after diagnosis for a further semen sample.

\section{Data analysis}

Data analysis was performed with the following tests: Kruskall-Wallis to compare the four separate groups of clinic volunteers in respect of age and semen findings; Fisher's exact as above for alcohol consumption and ethnic origin; Mann-Whitney $U$ (MWU) to compare semen findings in the different patient groups; $\chi^{2}$ for percentage of abnormal forms and percentage of non-progressors, McNemar's and the sign test for comparisons before and after treatment and standard non-parametric tests using SPSS (V.17.0; SPSS Inc).

\section{RESULTS}

One hundred and fifty-one clinic volunteers were recruited to the study. Thirty-four of these patients were subsequently found to have $C$ trachomatis and were therefore excluded from this analysis, leaving 117 patients whose data are presented here (data incomplete for one patient). These comprised 27 patients with asymptomatic NSU, 19 patients with symptomatic NSU, 64 clinic controls and seven patients who, despite symptoms of dysuria and/or discharge, were shown not to have NSU. In addition, the GP control group described above comprised 417 patients (data incomplete for one patient).

\section{Results of tests at presentation}

There were no significant differences between the four clinic groups in respect of age, excessive alcohol consumption (>28 units/week), ethnic origin (83 Caucasian and 33 nonCaucasian) and current smoking habit (table 1). There was a statistical difference between the four groups in respect of having a history of sexually transmitted infections, with the group of symptomatic non-NSU patients having the highest rate, $\mathrm{p}=0.007$ on MWU (StatXact software; Cytel, Cambridge, Massachusetts, UK). The overall prevalence of smoking, 34/116, $29 \%$ (95\% CI $21.0 \%$ to $37.6 \%$ ), compares with $22 \%$ among men in the UK in $2009 .{ }^{16}$

The population of the above clinic volunteers were shown to have statistically significantly worse total sperm counts and volume of semen compared with GP controls, $p=0.002$ and $p<0.001$, respectively, on $\mathrm{MWU}$. The finding for percentage of abnormal forms of $\mathrm{p}=0.04$ is not thought to be significant, given the large number of analyses performed. There were no significant differences in respect of motility, sperm concentration and percentage of non-progressors (table 2). The total sperm counts in the five different groups are shown in the figure 1.

Of the GP controls, 68/416 (16.3\%) had low sperm counts (<39 million) compared with $28 / 117$ (23.9\%) of clinic

Table 1 Demographic features of clinic attendees

\begin{tabular}{lllll}
\hline & $\begin{array}{l}\text { Controls } \\
(\mathbf{n = 6 3 )}\end{array}$ & $\begin{array}{l}\text { Asymptomatic } \\
\text { NSU (n=19) }\end{array}$ & $\begin{array}{l}\text { Symptomatic } \\
\text { NSU (n=27) }\end{array}$ & $\begin{array}{l}\text { Symptomatic } \\
\text { non-NSU (n=7) }\end{array}$ \\
\hline Age, mean (SD) & 27.6 & $26.7(7.4)$ & $28.7(5.8)$ & $25.9(5.2)$ \\
& $(7.1)$ & & & \\
Smoker (\%) & 25 & 33 & 42 & 14 \\
Excess alcohol (\%) & 24 & 19 & 16 & 14 \\
Ethnicity white (\%) & 73 & 70 & 84 & 57 \\
No history of STI & 64 & 52 & 42 & 14 \\
\hline
\end{tabular}

NSU, non-specific urethritis; STI, sexually transmitted infection. 
Table 2 Volume, sperm count and motility in clinic volunteers and GP controls

\begin{tabular}{|c|c|c|c|c|c|}
\hline & \multirow{2}{*}{$\begin{array}{l}N \text { (missing), } \\
N=534\end{array}$} & \multicolumn{3}{|c|}{ Quartiles } & \multirow{2}{*}{$\begin{array}{l}\text { MWU } \\
\text { p Value }\end{array}$} \\
\hline & & 25 & 50 & 75 & \\
\hline \multicolumn{6}{|l|}{ Volume } \\
\hline GU clinic & $117(0)$ & 1.8 & 2.4 & 3.4 & \multirow[t]{2}{*}{$<0.001$} \\
\hline GP & $417(0)$ & 2.2 & 3.2 & 4.4 & \\
\hline \multicolumn{6}{|l|}{ Sperm count } \\
\hline GU clinic & $117(0)$ & 47 & 108 & 200 & \multirow[t]{2}{*}{0.002} \\
\hline GP & $416(1)$ & 83 & 178 & 328 & \\
\hline \multicolumn{6}{|l|}{ Motility } \\
\hline GU clinic & $117(0)$ & 61 & 72 & 81 & \multirow[t]{2}{*}{0.55} \\
\hline GP & 398 (19) & 63 & 74 & 80 & \\
\hline \multicolumn{6}{|c|}{ Sperm concentration } \\
\hline GU clinic & $117(0)$ & 22 & 49 & 88 & \multirow[t]{2}{*}{0.25} \\
\hline GP & $417(0)$ & 32 & 60 & 94 & \\
\hline \multicolumn{6}{|c|}{ Percentage of abnormal forms } \\
\hline GU clinic & $117(0)$ & 86 & 88 & 89 & \multirow[t]{2}{*}{0.04} \\
\hline GP & $417(0)$ & 86 & 87 & 89 & \\
\hline \multicolumn{6}{|c|}{ Percentage of non-progressors } \\
\hline GU clinic & $117(0)$ & 12 & 19 & 26 & \multirow[t]{2}{*}{0.56} \\
\hline GP & $417(0)$ & 13 & 19 & 25 & \\
\hline
\end{tabular}

Volume in millilitres, total sperm count in millions, motility as percentage and sperm concentration as number per millilitre.

$\mathrm{GP}$, general practice; GU, genitourinary; N, number of patients (varies in GP group because of non-suitability of some specimens); MWU, Mann-Whitney $U$ test.

volunteers, Fisher's exact $\mathrm{p}=0.076$, with an OR (95\% CI) of 1.61 (0.98 to 2.65). This observed difference corresponded to a standardised difference of 0.33 and a post hoc power calculation confirmed that sample sizes were adequate after allowing both for multiple outcomes (by using 0.01 level of significance) and an imbalanced ratio of GP controls to clinic attendees (taken as four). The power to detect an effect size characterised by a standardised difference of 0.33 is just over $80 \%$ when using the sample sizes of 400 versus 100 , both somewhat below actual sample sizes employed in this study. Results are as follows when different thresholds are used. For instance, using above and below 100 million, the rates were GU 49.6\% versus GP $34.1 \%$,

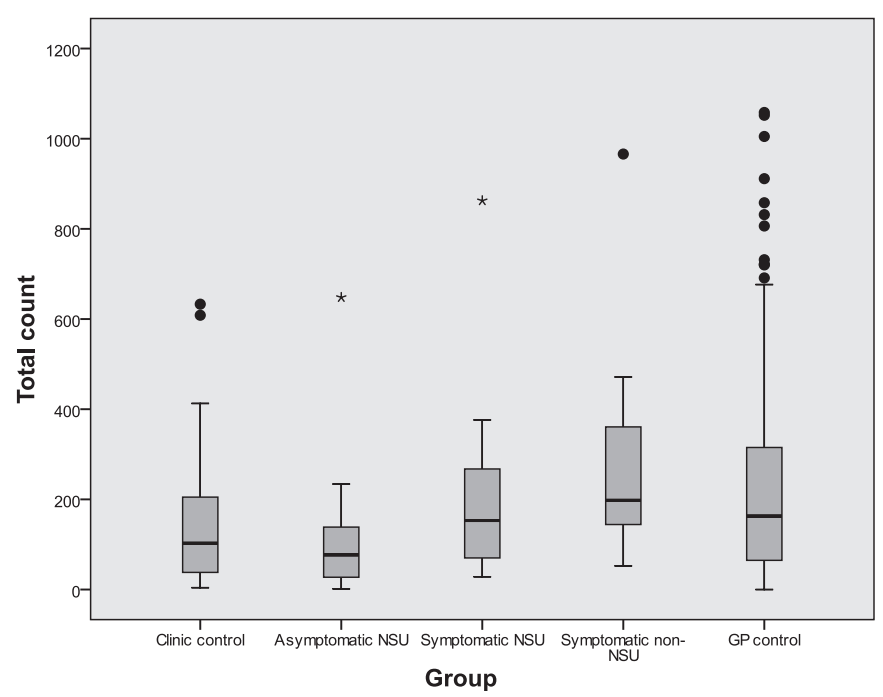

Figure 1 Total sperm counts in all groups in study. Number of clinic controls $=64$, asymptomatic non-specific urethritis (NSU) $=27$, symptomatic NSU $=19$, symptomatic non-NSU $=7$, general practice (GP) controls $=417$. Total sperm count in millions. Circles, outliers (>1.5 IQRs above 75th percentile). Stars, most extreme outliers. $\mathrm{p}=0.003$, OR 1.90 (1.25 to 2.87). Using a cut-point of 200 million, corresponding figures were $75.2 \%$ versus $57.9 \%$, $\mathrm{p}=0.001$, OR 2.20 (1.39 to 3.50). The total sperm counts in the different groups are shown in the figure 1. There were no statistical differences between these groups when comparing sperm concentration, motility or percentage of non-progressors.

Examining the four separate groups of volunteers (symptomatic NSU, asymptomatic NSU, clinic controls and symptomatic non-NSU patients), there was a statistical differences in total sperm counts between the four groups, $p=0.015$ on Kruskall-Wallis (table 3). There were no statistical differences between these groups when comparing volume, percentage of sperm concentration, motility, abnormal forms, percentage of non-progressors and white blood cells (Leucoscreen Test, performed in the 25 patients with five or more round cells per millilitre). Overall, 28/117 (23.9\%) clinic volunteers had low sperm counts (<39 million): 9/27 asymptomatic NSU, 3/19 symptomatic NSU, 16/64 clinic controls and 0/7 symptomatic non-NSU patients.

Compared with the rest of the clinic volunteers, asymptomatic NSU patients had statistically significantly lower total sperm counts $(p<0.02)$ on MWU but not significantly lower volumes, sperm concentration, motility, percentage of abnormal forms or percentage of non-progressors.

Asymptomatic NSU patients had statistically significantly lower total sperm counts compared with symptomatic NSU

Table 3 Volume, sperm count and motility in the four groups of clinic volunteers

\begin{tabular}{|c|c|c|c|c|c|}
\hline & \multirow[b]{2}{*}{$\mathbf{N}$} & \multicolumn{3}{|c|}{ Quartiles } & \multirow{2}{*}{$\begin{array}{l}K-W / \chi^{2} \\
\text { p Value }\end{array}$} \\
\hline & & 25 & 50 & 75 & \\
\hline \multicolumn{6}{|l|}{ Volume } \\
\hline Controls & 64 & 1.7 & 2.4 & 3.3 & \multirow[t]{4}{*}{0.29} \\
\hline Asymptomatic NSU & 27 & 1.4 & 2.0 & 3.2 & \\
\hline Symptomatic NSU & 19 & 2.1 & 3.0 & 3.7 & \\
\hline Symptomatic non-NSU & 7 & 2.2 & 2.4 & 3.4 & \\
\hline \multicolumn{6}{|l|}{ Sperm count } \\
\hline Controls & 64 & 45 & 108 & 205 & \multirow[t]{4}{*}{0.015} \\
\hline Asymptomatic NSU & 27 & 33 & 77 & 143 & \\
\hline Symptomatic NSU & 19 & 70 & 153 & 267 & \\
\hline Symptomatic non-NSU & 7 & 144 & 198 & 361 & \\
\hline \multicolumn{6}{|l|}{ Motility } \\
\hline Controls & 64 & 58 & 72 & 80 & \multirow[t]{4}{*}{0.11} \\
\hline Asymptomatic NSU & 27 & 64 & 72 & 81 & \\
\hline Symptomatic NSU & 19 & 55 & 67 & 82 & \\
\hline Symptomatic non-NSU & 7 & 79 & 81 & 83 & \\
\hline \multicolumn{6}{|l|}{ Sperm concentration } \\
\hline Controls & 64 & 21 & 49 & 85 & \multirow[t]{4}{*}{0.11} \\
\hline Asymptomatic NSU & 27 & 13 & 37 & 60 & \\
\hline Symptomatic NSU & 19 & 20 & 47 & 121 & \\
\hline Symptomatic non-NSU & 7 & 51 & 83 & 114 & \\
\hline \multicolumn{6}{|c|}{ Percentage of abnormal forms } \\
\hline Controls & 64 & 86 & 88 & 89 & \multirow[t]{4}{*}{0.18} \\
\hline Asymptomatic NSU & 27 & 87 & 88 & 89 & \\
\hline Symptomatic NSU & 19 & 87 & 89 & 90 & \\
\hline Symptomatic non-NSU & 7 & 86 & 87 & 87 & \\
\hline \multicolumn{6}{|c|}{ Percentage of non-progressors } \\
\hline Controls & 64 & 15 & 19 & 27 & \multirow[t]{4}{*}{0.41} \\
\hline Asymptomatic NSU & 27 & 12 & 20 & 27 & \\
\hline Symptomatic NSU & 19 & 10 & 16 & 21 & \\
\hline Symptomatic non-NSU & 7 & 7 & 16 & 27 & \\
\hline
\end{tabular}

Volume in millilitres, total sperm count in millions, motility as percentage and sperm concentration as number per millilitre.

NSU, non-specific urethritis; N, number of patients; $\mathrm{K}-\mathrm{W}$, Kruskall-Wallis test, used for top four parameters; $\chi^{2}$, used for bottom two parameters. 
patients $(p<0.02)$ on MWU but no differences in volume, sperm concentration, motility, percentage of abnormal forms or percentage of non-progressors.

Compared with GP controls, clinic controls had statistically significantly inferior total sperm counts $(p=0.009)$ and volume $(\mathrm{p}<0.001)$ on MWU but not sperm concentration, motility, percentage of abnormal forms or percentage of non-progressors.

\section{Results of follow-up tests}

Thirty-eight patients with NSU (17 asymptomatic and 21 symptomatic) returned for at least one follow-up test (38/42, $83 \%$ ). Comparisons were made between the various parameters in semen obtained at the first and last visits. The median time interval between the first and last semen specimens was 86 days (IOR=100 days).

Twelve of the 38 (three symptomatic and nine asymptomatic) had low total sperm counts at presentation. Eleven of these counts improved at follow-up ( $p=0.003$ on sign test). Eight of these achieved counts within the normal range.

McNemar's test on all 38 patients with NSU with repeat data shows no significant difference in whether or not the total counts were above or below the lower limit of normal (39 million) comparing semen at presentation and following resolution of the urethritis $(p=0.581)$. Neither were there significant differences in the other parameters compared.

\section{DISCUSSION}

Genitourinary (GU) medicine clinic attendees have been shown in this study to have statistically significantly inferior sperm counts, semen volume and percentage of abnormal forms compared with patients attending GP requesting a first check for possible infertility. This may be interpreted as showing that clinic attendees are more likely than the general population to carry organisms asymptomatically and that such organisms are potentially damaging to male fertility. These are organisms that are hitherto unknown or not tested for in routine practice. GU clinic attendees are known to be at high risk of carrying pathogens asymptomatically, for example, $43 \%$ of women attending this GU clinic were shown to have oncogenic human papillomavirus. ${ }^{17}$ The decision to use the GP controls was taken after it was realised that the clinic controls had a high rate of semen abnormalities, and our attempts to recruit a community control group had failed.

Second, patients with asymptomatic NSU have statistically significantly lower sperm counts compared with the rest of the clinic volunteers and when compared with patients with symptomatic NSU. The explanation for this may be as follows. Patients with NSU carry organisms that can cause inflammation. If such a patient has symptoms, he is likely to present more quickly to a GU clinic than a patient with NSU who does not have symptoms. Therefore, organisms in the latter group will often have more time to damage the production of semen.

Third, even the seemingly normal clinic controls have lower sperm counts and semen volume than the GP controls. If asymptomatic organisms are the cause of the semen abnormalities in other clinic attendees and it is known that patients attending GU clinics are at high risk of harbouring pathogens asymptomatically, this may be interpreted as showing that clinic controls, with no symptoms or signs suggestive of infection, nevertheless are more likely to have organisms with the capacity to damage semen.

There is some evidence to indicate that $C$ trachomatis infection can affect sperm quality and sperm function ${ }^{18}$ including, in vitro studies that show that co-incubation of spermatozoa with $C$ trachomatis causes a significant decline in numbers of motile sperm and results in premature sperm death. ${ }^{19}$

In a large study, Mazzoli et $a l^{20}$ found strong associations in men with CT-positive prostatitis and low values for sperm concentration, percentage of motile sperm and normal morphological forms together with evidence for probable immunomediated damage to germinal cells. The same group had previously found anti-C trachomatis mucosal IgA in a high percentage of patients with prostatitis, suggesting that this organism could be causal in some cases. ${ }^{21}$

Ureaplasma biovar 2 (formerly Ureaplasma urealyticum), frequently found in the urethra, has been shown to damage spermatozoa. $^{22}{ }^{23}$ Mycoplasma genitalium, a common cause of urethritis, ${ }^{24}$ can cause immobility of spermatozoa in vitro. ${ }^{25} \mathrm{~N}$ gonorrhoeae is a less common cause of urethritis in the $\mathrm{UK}^{24}$ and although biologically plausible, it is currently unclear whether it can cause male factor infertility. ${ }^{26}$ An uncommon cause of urethritis, Trichomonas vaginalis, has been linked with male factor infertility. ${ }^{27}$

Recognised causes of NGU such as $C$ trachomatis and $M$ genitalium are known to cause asymptomatic infection at times. One study found this to be the case in $60 \%$ of cases of $C$ trachomatis urethritis and $27 \%$ of urethritis caused by $M$ genitalium. ${ }^{28}$

Finally, almost all the NSU patients with low sperm counts improved following treatment. On the other hand, those with normal counts did not increase. There are two possible explanations. This may merely represent regression to the mean of statistical outliers. Alternatively, it may be argued that those with normal counts have not had damage by organisms and therefore treatment of those organisms would not be expected to produce an increase. Only further research can clarify this issue.

In male fertility in general, the results of antibiotic treatment have been disappointing. Individual agents within each of the major classes of antibiotics have been shown to have significant adverse effects on spermatogenesis or spermatozoal function

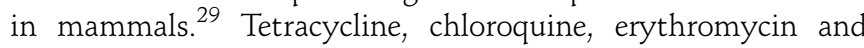
co-trimoxazole considerably impair sperm movement characteristics and significantly reduce sperm viability in vitro. ${ }^{30}$ Amoxicillin appears to have negligible effect. ${ }^{30}$ Furthermore, a controlled trial showed no benefit on semen quality from the use of erythromycin. ${ }^{31}$ The patients attending GU clinics, with or without NSU, may, however, be a special case, given that their semen abnormalities are often likely to be caused by infection and therefore abnormalities may be more likely to improve with antibiotics.

In this study, the timing of the semen sample after previous ejaculation was asked routinely of clinic volunteers and those not meeting the accepted criteria were not invited to join the study. A possible weakness of the study is that it cannot be known whether the GP comparator group included some who did not meet these criteria. However, if so, such patients would be expected to have had lower quality semen as a result. This would have had the effect of reducing the apparent differences in semen between clinic volunteers and GP Controls. It is possible therefore that the differences demonstrated in this study between clinic and GP attendees would have been even greater if the guidance on timing of the samples had always been strictly adhered to by GP controls. Furthermore, if it had been possible to recruit a genuine cross-section of the general public, it is presumed that the differences would be substantially greater. The authors expect that the results could be generalised to other populations attending Sexual Health clinics.

The findings of this study raise several questions. First, is the carriage of unknown organisms responsible for the high rate of 


\section{Key messages}

- GU clinic attendees are more likely to have abnormalities of semen than patients attending GP for a first check for possible infertility.

- High rates of abnormal semen findings are found in patients with and without NSU but the highest rate occurred in those with asymptomatic NSU.

- The question of whether or not antibiotic treatment improves low sperm counts in patients with NSU requires further research.

various semen abnormalities found in GU clinic attendees? Second, does the finding that, among clinic attendees, patients with asymptomatic NSU are most likely to have low sperm counts imply that this condition is pathogenic and, like symptomatic NSU, therefore requires treatment? Third, is the high rate of semen abnormalities in clinic controls indicative of the silent carriage of unknown organisms in some attendees in this group? This study also points to the need for further research, in particular to resolve whether or not antibiotic treatment improves low sperm counts in patients with NSU.

Acknowledgements The authors thank Hamid Jalal, Tom Fanshawe, Chris Sonnex, Susie Forster, Andrew Prentice, Raj Mathur and Sandy Paget for their help in planning the project. Raj Mathur for commenting on a draft of the paper. Julia Burdett and Joanne Gibbs for their help in recruiting volunteers.

Contributors Roles of authors CAC: design of project, recruitment of patients, completion of clinical questionnaires, analysis of results; principal writer SC: design of project, cytological examinations and creation of cytological spreadsheet; co-writer CP: statistical analysis; co-writer OG: cytological examinations; co-writer SB: completion of clinical questionnaires and creation of spreadsheet; co-writer RW: cytological examinations; co-writer AG-M: completion of clinical questionnaires and creation of clinical spreadsheet; co-writer MO'D: design of project and co-writer.

Competing interests None.

Ethics approval Ethics approval was provided by Peterborough and Fenland LREC

Provenance and peer review Not commissioned; externally peer reviewed.

\section{REFERENCES}

1. Williams C, Giannopoulos T, Sherriff EA. Investigation of infertility with the emphasis on laboratory testing and with reference to radiological imaging. J Clin Pathol 2003; $56: 261-7$

2. Hirsh A. Male subfertility. BMJ 2003;327:669-72.

3. Younglai EV, Holloway AC, Foster WG. Environmental and occupational factors affecting fertility and IVF success. Hum Reprod Update 2005;11:43-57.

4. STI Annual Data Tables. STI Table 2a. Country UK. 2009. http://www.hpa.org.uk/ web/HPAWebFile/HPAweb C/1215589013908 (accessed 13 May 2011).

5. Carey AJ, Beagley KW. Chlamydia trachomatis, a hidden epidemic: effects on female reproduction and options for treatment. Am J Reprod Immunol 2010:63:576-86.
6. Dieterle S. Urogenital infections in reproductive medicine. Andrologia 2008;40:117-19

7. Greendale GA, Haas ST, Holbrook K, et al. The relationship of Chlamydia trachomatis infection and male infertility. Am J Public Health 1993:83:996-1001.

8. McKay L, Clery H, Carrick-Anderson K, et al. Genital Chlamydia trachomatis infection in a subgroup of young men in the UK. Lancet 2003:361:1792.

9. Grosskurth H, Mayaud P, Mosha F, et al. Asymptomatic gonorrhoea and chlamydial infection in rural Tanzanian men. BMJ 1996;312:277.

10. Mandal D, Gillanders V. Chlamydial infection is asymptomatic in England as well as Tanzania. BMJ 1996;312:1231

11. Comhaire FH, Mahmoud AMA, Depuydt CE, et al. Mechanisms and effects of male genital tract infection on sperm quality and fertilising potential: the andrologist's viewpoint. Hum Reprod Update 1999;5:393-8.

12. Clinical Effectiveness Group, British Association for Sexual Health and HIV. UK National Guideline on the Management of Non-gonococcal Urethritis, 2007. http:// www.bashh.org/guidelines

13. Horner PJ. Should we still be testing for asymptomatic non-specific urethritis in departments of genitourinary medicine? Int J STD AIDS 2005;16:273-7.

14. Schiefer MG. Microbiology of male urethroadnexitis: diagnostic procedures and criteria for aetiological classification. Andrologia 1998:30:7-13.

15. WHO Laboratory Manual For The Examination And Processing Of Human Semen. 5th edn. 2010. http://www.who.int/reproductivehealth/publications/infertility/ 9789241547789/en/index.html

16. Cancer Research UK-Smoking statistics. 2009. http://info.cancerresearchuk.org/ cancerstats/types/lung/smoking/\#source9

17. Jalal H, Stephen $H$, Bibby DF, et al. Molecular epidemiology of genital human papillomavirus and Chlamydia trachomatis among patients attending a genitourinary medicine clinic-will vaccines protect? Int J STD AIDS 2007; 18:617-21.

18. Akande V, Turner $C$, Horner $P$, et al. Impact of Chlamydia trachomatis in the reproductive setting: British fertility society guidelines for practice. Hum Fertil (Camb) 2010;13:115-25

19. Eley A, Pacey AA, Galdiero M, et al. Can Chlamydia trachomatis directly damage your sperm? Lancet Infect Dis 2005:5:53-7.

20. Mazzoli S, Cai T, Addonisio P, et al. Chlamydia trachomatis infection is related to poor sperm quality in young prostatitis patients. Eur Urol 2010;57:708-14.

21. Mazzoli S, Cai T, Rupealta V, et al. Interleukin 8 and anti-chlamydia trachomatis mucosal $\lg A$ as urogenital immunologic markers in patients with $C$ trachomatis prostatic infection. Eur Urol Eur Urol 2007:51:1385-93.

22. Nunez-Calonge $\mathbf{R}$, Cabellero $\mathrm{P}$, Redondo C, et al. Ureaplasma urealyticum reduces motility and induces membrane alterations in human spermatozoa. Hum Reprod 1998:13:2756-61.

23. Reichart M, Kahane I, Bartoov B. In vivo and in vitro impairment of human and ram sperm nuclear chromatin integrity by sexually transmitted Ureaplasma urealyticum infection. Biol Reprod 2000;63:1041-8.

24. Taylor-Robinson D, Horner PJ. The role of Mycoplasma genitalium in nongonococcal urethritis. Sex Transm Infect 2001;77:229-31.

25. Svenstrup HF, Fedder J, Abraham-Peskir J, et al. Mycoplasma genitalium attaches to human spermatozoa. Hum Rep 2003;18:2103-9.

26. Ness RB, Markovic N, Carlson CL, et al. Do men become infertile after having sexually transmitted urethritis? An epidemiologic examination. Fertil Steril 1997;68:205-13.

27. Soper D. Trichomoniasis: under control or undercontrolled? Am J Obstet Gynecol 2004; 190:281-90.

28. Falk L, Fredlund H, Jensen JS. Symptomatic urethritis is more prevalent in men infected with Mycoplasma genitalium than with Chlamydia trachomatis. Sex Transm Infect 2004;80:289-93.

29. Schlegel PN, Chang TS, Marshall FF. Antibiotics: potential hazards to male fertility. Fertile Steril 1991:55:235-42.

30. Hargreaves CA, Rogers S, Hills F, et al. Effects of co-trimoxazole, erythromycin, amoxycillin, tetracycline and chloroquine on sperm function in vitro. Hum Reprod 1998;13:1878-86.

31. Baker HW, Straffon WG, McGowan MP, et al. A controlled trial of the use of erythromycin for men with asthenospermia. Int J Androl 1984;7:383-8. 